\title{
Quantum Monte Carlo Analysis of Exchange and Correlation in the Strongly Inhomogeneous Electron Gas
}

\author{
Maziar Nekovee, ${ }^{1, *}$ W. M. C. Foulkes,${ }^{1}$ and R. J. Needs ${ }^{2}$ \\ ${ }^{1}$ The Blackett Laboratory, Imperial College, Prince Consort Road, London SW7 2BZ, United Kingdom \\ ${ }^{2}$ Cavendish Laboratory, Madingley Road, Cambridge CB3 OHE, United Kingdom
}

(Received 31 July 2000; published 25 June 2001)

\begin{abstract}
We use the variational quantum Monte Carlo method to calculate the density-functional exchangecorrelation hole $n_{\mathrm{xc}}$, the exchange-correlation energy density $e_{\mathrm{xc}}$, and the total exchange-correlation energy $E_{\mathrm{xc}}$ of several strongly inhomogeneous electron gas systems. We compare our results with the local density approximation and the generalized gradient approximation. It is found that the nonlocal contributions to $e_{\mathrm{xc}}$ contain an energetically significant component, the magnitude, shape, and sign of which are controlled by the Laplacian of the electron density.
\end{abstract}

DOI: 10.1103/PhysRevLett.87.036401

PACS numbers: 71.15.Mb, 71.10.-w, 71.45.Gm

The Kohn-Sham density-functional theory (DFT) [1] shows that it is possible to calculate the ground-state properties of interacting many-electron systems by solving only one-electron Schrödinger-like equations. The results are exact in principle, but in practice it is necessary to approximate the unknown exchange-correlation (XC) energy functional, $E_{\mathrm{xc}}[n]$, which expresses the many-body effects in terms of the electron density $n(\mathbf{r})$. The current popularity of density-functional methods in condensed matter physics, quantum chemistry, and materials science reflects the remarkable success of fairly simple approximate XC energy functionals.

In the local density approximation (LDA), the $\mathrm{XC}$ hole $n_{\mathrm{xc}}\left(\mathbf{r}, \mathbf{r}^{\prime}\right)$ about an electron at $\mathbf{r}$ is approximated by the $\mathrm{XC}$ hole of a uniform electron gas of density $n=n(\mathbf{r})$. This works surprisingly well, but not well enough for many chemical and biological applications. The most widely used correction to the LDA is the generalized gradient approximation (GGA) [2-4], in which the effects of inhomogeneity are modeled using the semilocal approximation $E_{\mathrm{xc}} \approx \int d \mathbf{r} f(n,|\nabla n|)$, where $f$ is some parametrized nonlinear function of $n$ and $\nabla n$. A common feature of all current GGAs is that their construction is guided by limiting behaviors and sum rules; they are designed to fit various integrated quantities such as total exchange energies of atoms or ionization energies of molecules, but incorporate little or no information about the behavior of local quantities such as $n_{\mathrm{xc}}\left(\mathbf{r}, \mathbf{r}^{\prime}\right)$ and the exchange-correlation energy density $e_{\mathrm{xc}}(\mathbf{r})$ in strongly inhomogeneous systems. This may explain why current GGAs, although better than the LDA in many situations, are not consistently able to deliver the very high accuracy of $\sim 0.1 \mathrm{eV}$ required to study, e.g., most chemical reactions. To open a new direction in the search for improved functionals, we use the variational quantum Monte Carlo (VMC) method [5] to calculate $n_{\mathrm{xc}}$ and $e_{\mathrm{xc}}$ for several strongly inhomogeneous electron gases and analyze the performance of the LDA and the GGA in these systems in detail.

The inhomogeneous electron gases considered all had the same average electron density $n^{0}=3 /\left(4 \pi r_{s}^{3}\right)$, where $r_{s}=2 a_{0}$ (approximately the same as for $\mathrm{Al}$ ). The strong density modulations were one dimensional and periodic, with a roughly sinusoidal profile. Three different modulation wave vectors $q \leq 2.17 k_{F}^{0}$ were investigated, where $k_{F}^{0}$ is the Fermi wave vector corresponding to $n^{0}$. The strong variation of $n(\mathbf{r})$ on the scale of the inverse local Fermi wave vector $k_{F}(\mathbf{r})^{-1}=\left[3 \pi^{2} n(\mathbf{r})\right]^{-1 / 3}$ results in a strikingly nonlocal behavior of $n_{\mathrm{xc}}$ that cannot be described by semilocal corrections to the LDA. We show, however, that the LDA errors in $e_{\mathrm{xc}}$ have a dominant and energetically significant component, the magnitude, shape, and sign of which are controlled by the semilocal quantity $\nabla^{2} n(\mathbf{r})$. Because it depends only on $n$ and $|\nabla n|$, the GGA is unable to correct the LDA errors in $E_{\mathrm{xc}}$ resulting from this component adequately, and worsens the LDA in two of our three systems. The relevance of Laplacian terms has been pointed out previously [6], but our calculations provide the first quantitative evidence of their importance in strongly inhomogeneous systems and for $e_{\mathrm{xc}}$.

Our starting point is the adiabatic connection formula [7], which expresses $E_{\mathrm{xc}}[n]$ as the volume integral of an $\mathrm{XC}$ energy density $e_{\mathrm{xc}}$ defined by [8]

$$
e_{\mathrm{xc}}(\mathbf{r},[n])=\frac{1}{2} \int d \mathbf{r}^{\prime} \frac{n(\mathbf{r}) n_{\mathrm{xc}}\left(\mathbf{r}, \mathbf{r}^{\prime}\right)}{\left|\mathbf{r}-\mathbf{r}^{\prime}\right|},
$$

where $n_{\mathrm{xc}}$ is the coupling-constant-averaged XC hole. The definition of $e_{\mathrm{xc}}$ used in the construction of the GGA differs from ours by an integration by parts that does not affect the integrated $E_{\mathrm{xc}}$. We favor Eq. (1), however, because of its clear physical interpretation and because it aids comparison with the LDA XC energy density, which is constructed from an approximate hole.

The XC hole $n_{\mathrm{xc}}$ is obtained via a coupling-constant integration,

$$
\begin{aligned}
n(\mathbf{r}) n\left(\mathbf{r}^{\prime}\right)+ & n(\mathbf{r}) n_{\mathrm{xc}}\left(\mathbf{r}, \mathbf{r}^{\prime}\right) \\
= & \int_{0}^{1} d \lambda\left\langle\Psi^{\lambda}\left|\sum_{i} \sum_{j(\neq i)} \delta\left(\mathbf{r}-\mathbf{r}_{i}\right) \delta\left(\mathbf{r}^{\prime}-\mathbf{r}_{j}\right)\right| \Psi^{\lambda}\right\rangle,
\end{aligned}
$$

where $\Psi^{\lambda}$ is the antisymmetric ground state of the Hamiltonian $\hat{H}^{\lambda}=\hat{T}+\lambda \hat{V}_{e e}+\hat{V}^{\lambda}$ associated with coupling 
constant $\lambda$. Here $\hat{T}$ and $\hat{V}_{e e}$ are the operators for the kinetic and electron-electron interaction energies, and $\hat{V}^{\lambda}=$ $\Sigma V^{\lambda}\left(\mathbf{r}_{i}\right)$ is the one-electron potential needed to hold the electron density $n^{\lambda}(\mathbf{r})$ associated with $\Psi^{\lambda}$ equal to $n(\mathbf{r})$ for all values of $\lambda$ between 0 and 1. Our VMC method [9] for calculating $n_{\mathrm{xc}}$ and $e_{\mathrm{xc}}$ from Eqs. (1) and (2) is a generalization of the scheme used by Hood et al. [10]. It amounts to treating both $\Psi^{\lambda}$ and $V^{\lambda}$ variationally and determining the variational parameters by simultaneously minimizing the variance of the local energy [11] and the deviation of $n^{\lambda}$ from $n$ [9].

The VMC simulations were performed for a finite spinunpolarized electron gas in a face-centered cubic simulation cell subject to periodic boundary conditions. The exact interacting ground-state density $n(\mathbf{r})$ was chosen a priori, and the constrained minimization scheme was then used to find, at each $\lambda$, the exact (within VMC) wave function $\Psi^{\lambda}$ and external potential $V^{\lambda}$ corresponding to that density [12]. At full coupling $(\lambda=1), V^{\lambda}(\mathbf{r})$ is the exact external potential of the many-electron system with ground-state density $n(\mathbf{r})$. The input density $n(\mathbf{r})$ was generated by solving, within the LDA, the Kohn-Sham equations for an external potential of the form $V_{q} \cos (\mathbf{q} \cdot \mathbf{r})$, where $V_{q}$ was fixed at $2.08 \epsilon_{F}^{0}$ and $\epsilon_{F}^{0}$ is the Fermi energy corresponding to $n^{0}$. The advantages of this procedure are that the input electron density is guaranteed to be noninteracting v-representable and that the Slater determinant of single-particle orbitals is by construction the exact many-body wave function corresponding to $\lambda=0$. The (density-functional) exchange contributions to $e_{\mathrm{xc}}, n_{\mathrm{xc}}$, and $E_{\mathrm{xc}}$, obtained from this Slater determinant, are therefore also exact [9].

We consider potentials with $q=1.11 k_{F}^{0}, 1.55 k_{F}^{0}$, and $2.17 k_{F}^{0}$, and cells containing 64, 78, and 69 electrons, respectively. The adiabatic calculations were performed using six equidistant values of $\lambda$ in the range $[0,1]$ and with the Slater-Jastrow ansatz given in [9] as our many-body wave function. At each $\lambda$, we used a total of 20 variational parameters in $\Psi^{\lambda}$ and up to seven coefficients in the plane-wave expansions of $n^{\lambda}$ and $V^{\lambda}$. The optimization of the parameters in $\Psi^{\lambda}$ and $V^{\lambda}$ was performed using 96000 statistically uncorrelated electron configurations. This was sufficient to reduce the root mean square deviation of $n^{\lambda}(\mathbf{r})$ from $n(\mathbf{r})$ to less than $0.5 \%$ of $n(\mathbf{r})$ for all values of $\lambda$ and all systems. The expectation values were calculated [5] using $10^{6}$ independent configurations of all electrons. Throughout, we used the modified electronelectron interaction described in [13], which virtually eliminates the finite-size errors arising from the long range of the Coulomb potential. The statistical errors were negligible except in $n_{\mathrm{xc}}$ in low-density regions, where they were much smaller than the differences between $n_{\mathrm{xc}}$ and $n_{\mathrm{xc}}^{\mathrm{LDA}}$. The largest systematic errors are caused by the finite size of the system and the approximate nature of $\Psi^{\lambda}$. These errors combine such that, even in a homogeneous electron gas, $e_{\mathrm{xc}}^{\mathrm{VMC}} \neq e_{\mathrm{xc}}^{\mathrm{LDA}}$. To circumvent this problem, we performed additional VMC calculations of the XC energies of finite homogeneous electron gases with $N=64$ and $r_{s}=0.8,1,2,3,4,5,8$, and 10 . This enabled us to construct a Perdew-Zunger parametrization [14] of the VMC XC energy per electron of a finite uniform electron gas with $N=64$. By using this parameterization to calculate $e_{\mathrm{xc}}^{\mathrm{LDA}}$ in all systems studied, we largely eliminated the systematic errors in the calculated differences between $e_{\mathrm{xc}}^{\mathrm{VMC}}$ and $e_{\mathrm{xc}}^{\mathrm{LDA}}$. The same parametrization was also used as input for the evaluation of $E_{\mathrm{xc}}^{\mathrm{GGA}}$ [2], a procedure that we assume mitigates the errors in $E_{\mathrm{xc}}^{\mathrm{GGA}}-E_{\mathrm{xc}}^{\mathrm{VMC}}$.

In Fig. 1 we show snapshots of the deformation of $n_{\mathrm{xc}}^{\mathrm{VMC}}$ around an electron moving in the $q=1.11 k_{F}^{0}$ system along a line parallel to $\mathbf{q}$, the direction of maximum inhomogeneity, from a density maximum towards the tail of $n(\mathbf{r})$. The XC hole is plotted as a function of $\mathbf{r}^{\prime}$ around a fixed electron at $\mathbf{r}$, with $\mathbf{r}^{\prime}$ ranging in a plane parallel to q. Also shown is the corresponding LDA hole $n_{\mathrm{xc}}^{\mathrm{LDA}}$ [7]. At the density maximum (not shown), both $n_{\mathrm{xc}}^{\mathrm{VMC}}$ and $n_{\mathrm{xc}}^{\mathrm{LDA}}$ are centered at the electron. However, unlike $n_{\mathrm{xc}}^{\mathrm{LDA}}$, which is always spherically symmetric, $n_{\mathrm{xc}}^{\mathrm{VMC}}$ is contracted in the direction of the inhomogeneity. As the electron moves away from the density maximum to a point on the slope (top panel), the nonlocal nature of $n_{\mathrm{xc}}^{\mathrm{VMC}}$ becomes manifest. While $n_{\mathrm{xc}}^{\mathrm{LDA}}$ is still centered at the electron and is rather diffuse, $n_{\mathrm{xc}}^{\mathrm{VMC}}$ lags behind near the density maximum and is much more compact. The nonlocal behavior of $n_{\mathrm{xc}}^{\mathrm{VMC}}$ becomes remarkable at the density minimum. Here $n_{\mathrm{xc}}^{\mathrm{VMC}}$ has two large nonlocal minima, each centered at a density maximum $\sim 2.80$ a.u.
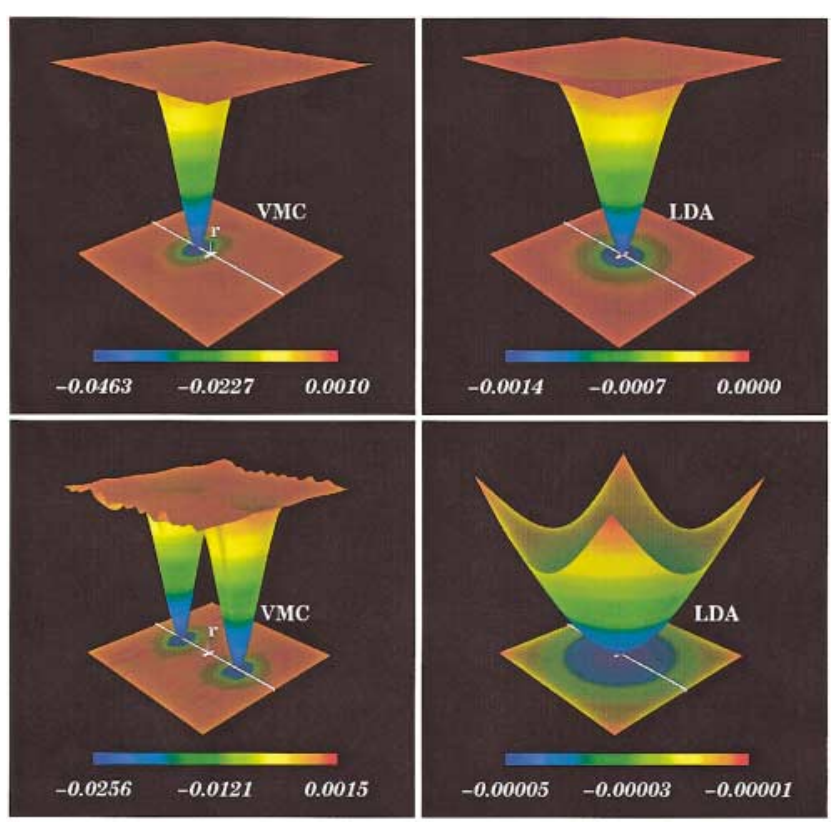

FIG. 1 (color). The VMC and LDA $n_{\mathrm{xc}}\left(\mathbf{r}, \mathbf{r}^{\prime}\right)$ for the $q=$ $1.11 k_{F}^{0}$ system plotted around an electron fixed at $\mathbf{r}$ (indicated in the figure), with $\mathbf{r}^{\prime}$ ranging in a plane parallel to $\mathbf{q}$ : (top) electron on the slope; (bottom) electron at a density minimum. The solid line shows the direction parallel to $\mathbf{q}$ (see Fig. 2 for the profile of the electron density along this line). 
away from the electron (the small fluctuations of $n_{\mathrm{xc}}^{\mathrm{VMC}}$ at this point are statistical errors). The LDA hole, by contrast, is spread over the whole system in order to satisfy the sum rule [7]: $\int d \mathbf{r}^{\prime} n_{\mathrm{xc}}^{\mathrm{LDA}}\left(\mathbf{r}, \mathbf{r}^{\prime}\right)=-1$. This striking nonlocality of $n_{\mathrm{xc}}^{\mathrm{VMC}}$ also occurs in the other two systems we considered, and similar nonlocal behavior has previously been observed for the exchange plus Coulomb hole of the hydrogen molecule [15]. Clearly, semilocal corrections are unable to significantly improve the LDA description of the XC hole in our systems, and fully nonlocal approximations are required. We found, however, that, despite the strong nonlocality of $n_{\mathrm{xc}}^{\mathrm{VMC}}$, the LDA errors in $e_{\mathrm{xc}}^{\mathrm{VMC}}$ can be described in terms of a semilocal quantity, the Laplacian of the electron density.

In Fig. 2 we show $e_{\mathrm{xc}}^{\mathrm{LDA}}-e_{\mathrm{xc}}^{\mathrm{VMC}}$ for two of the strongly inhomogeneous systems studied, where $e_{\mathrm{xc}}^{\mathrm{LDA}}$ is calculated using the exact ground-state density $n(\mathbf{r})$. (The very similar graph for the third system has been omitted to save space.) The results are plotted along a line parallel to $\mathbf{q}$ (we call this direction $y$ ). Also shown are $n(\mathbf{r})$ and $\nabla^{2} n(\mathbf{r})$ plotted along the same line. It is apparent that the shape, magnitude, and sign of the LDA errors in $e_{\mathrm{xc}}$ closely follow the shape, magnitude, and sign of $\nabla^{2} n(\mathbf{r})$. The LDA errors in $e_{\mathrm{xc}}$ are large and negative in regions where $\nabla^{2} n(\mathbf{r})$ is large and negative (around density maxi$\mathrm{ma})$, and large and positive in regions where $\nabla^{2} n(\mathbf{r})$ is large and positive. The GGA XC energy density is not defined via Eq. (1) and is not shown here [16]. The VMC values of the integrated $E_{\mathrm{xc}}$ are shown in Table I, along with the differences $\Delta E_{\mathrm{xc}}^{\mathrm{LDA}}=E_{\mathrm{xc}}^{\mathrm{LDA}}-E_{\mathrm{xc}}^{\mathrm{VMC}}$ and $\Delta E_{\mathrm{xc}}^{\mathrm{GGA}}=E_{\mathrm{xc}}^{\mathrm{GGA}}-E_{\mathrm{xc}}^{\mathrm{VMC}}$ (the version of the GGA used here is due to Perdew, Burke, and Ernzerhof [2]). The LDA errors in $E_{\mathrm{xc}}$ reflect the profound effect of the Laplacian errors in $e_{\mathrm{xc}}$ and change sign from positive (for the

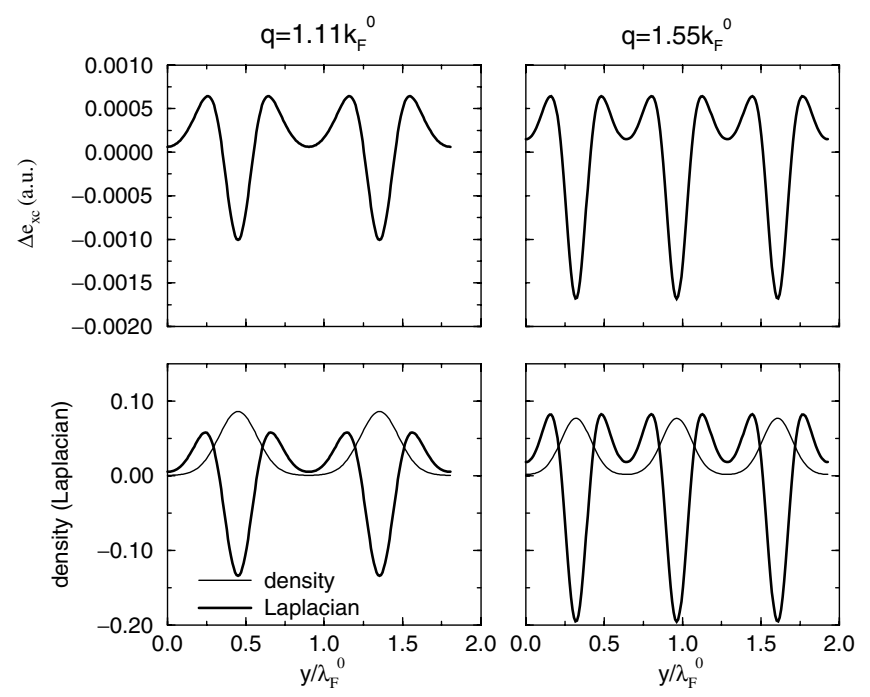

FIG. 2. The upper graphs show $e_{\mathrm{xc}}^{\mathrm{LDA}}-e_{\mathrm{xc}}^{\mathrm{VMC}}$ along a direction parallel to $\mathbf{q}$ for two different strongly inhomogeneous systems. The lower graphs show the corresponding electron densities (light lines) and Laplacians (heavy lines). Distances are in units of the Fermi wavelength $\lambda_{F}^{0}=2 \pi / k_{F}^{0}$. $q=1.11 k_{F}^{0}$ system) to negative (for the two other systems) as $q$ increases and the negative contributions to $\Delta e_{\mathrm{xc}}$, which occur where $\nabla^{2} n(\mathbf{r})<0$, become dominant. The GGA corrections are by construction always negative [2-4]; they improve $E_{\mathrm{xc}}^{\mathrm{LDA}}$ for the $q=1.11 k_{F}$ system but worsen it for the two other systems. We note that GGA corrections also worsen the LDA in quasi-two-dimensional electron gases [17], a shortcoming shared by the recently proposed meta-GGA (MGGA) [18]. Although our sytems are not quasi two dimensional [19], we believe that a similar semilocal effect lies behind the failure of the GGA in both cases, namely, an increasing negative contribution to the LDA errors in $e_{\mathrm{xc}}$ originating from the strongly negative Laplacian around the peak of $n(\mathbf{r})$. Similar errors are known to occur near the nuclei in molecules, but are overcompensated by positive errors in the bonding and outer regions [20]. We note that, since $\nabla^{2} n(\mathbf{r})$ integrates to zero, an energy-density correction proportional to $\nabla^{2} n(\mathbf{r})$ would not improve $E_{\mathrm{xc}}^{\mathrm{LDA}}$. As shown below, however, the dependence on $\nabla^{2} n$ observed here is nonlinear.

The exchange-correlation energy density is a unique functional of the electron density. Provided that the electron density has a convergent Taylor expansion about the point $\mathbf{r}$, it can therefore be written as $e_{\mathrm{xc}}(\mathbf{r},[n])=$ $e_{x c}\left[\mathbf{r}, n(\mathbf{r}), \nabla_{i} n(\mathbf{r}), \nabla_{i} \nabla_{j} n(\mathbf{r}), \ldots\right] . \quad$ The GGA may be viewed as an attempt to approximate this mapping by a nonlinear function of $n(\mathbf{r})$ and $\nabla_{i} n(\mathbf{r})$ only. In the case of the exchange energy (although not, unfortunately, the correlation energy), a uniform scaling argument [21] shows that $e_{x}=F_{x}(s, l, \ldots) e_{x}^{\mathrm{LDA}}$, where $e_{x}$ is the exact (density-functional) exchange-energy density obtained from $\Psi^{\lambda=0}$ [9], $F_{x}$ is an enhancement factor, $e_{x}^{\mathrm{LDA}}$ is the exchange-energy density within the LDA, and $s=|\nabla n| /\left[2 k_{F}(\mathbf{r}) n(\mathbf{r})\right]$ and $l=\nabla^{2} n /\left[4 k_{F}^{2}(\mathbf{r}) n(\mathbf{r})\right]$ are a dimensionless gradient and a dimensionless Laplacian, respectively. Figures $3 \mathrm{a}$ and $3 \mathrm{~b}$ are scatter plots comparing the values of $F_{x}$ at different points in space with the values of $s$ and $l$ at those points. Because $n(\mathbf{r})$ appears in the denominator of $l$ and $s$, the maximum absolute values of $l$ and $s$ occur where the electron density is smallest and are largest for the $q=1.11 k_{F}$ system.

The two-valued nature of Fig. 3a arises because the mapping from $s$ to position is two-valued in our systems. For example, $s$ is zero at both the minimum and the maximum of the density, where the required corrections to the LDA exchange hole are completely different, as can be inferred from Fig. 1 [22]. In sharp contrast, Fig. 3b shows

TABLE I. Exchange-correlation energies (Hartrees per electron) and the LDA and GGA errors in this quantity for different values of the wave vector $\mathbf{q}$. The statistical errors in $E_{\mathrm{xc}}^{\mathrm{VMC}}$ are indicated.

\begin{tabular}{cccc}
\hline \hline$q / k_{F}^{0}$ & $E_{\mathrm{xc}}^{\mathrm{VMC}}$ & $\Delta E_{\mathrm{xc}}^{\mathrm{LDA}}$ & $\Delta E_{\mathrm{xc}}^{\mathrm{GGA}}$ \\
\hline 1.11 & $-0.3289 \pm 0.001$ & +0.0042 & +0.0001 \\
1.55 & $-0.3127 \pm 0.001$ & -0.0005 & -0.0074 \\
2.17 & $-0.2882 \pm 0.001$ & -0.0066 & -0.0140 \\
\hline \hline
\end{tabular}

$036401-3$ 

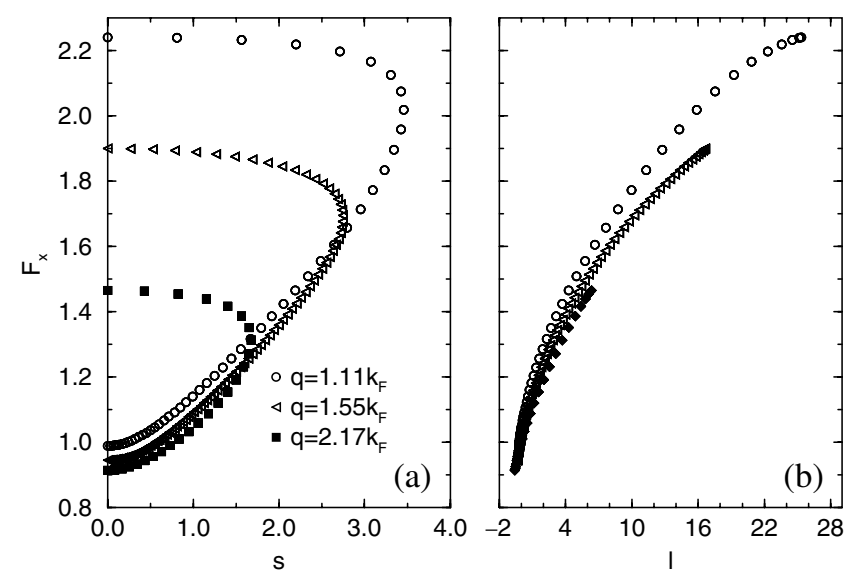

FIG. 3. The left panel (a) plots values of the exact exchange enhancement factor $F_{x}$ against values of the reduced density gradient for three strongly inhomogeneous systems. The right panel (b) plots values of the exact exchange enhancement against values of the reduced density Laplacian.

that $F_{x}$ is a simple and almost unique function of $l$, with the physically different regions around the maximum and the minimum of the density corresponding to regions of negative and positive Laplacian, respectively. This suggests that the inclusion of Laplacian terms may allow the construction of simpler and more accurate approximate functionals. Springborg and Dahl recently reached a similar conclusion [23] based on their studies of the exchangeenergy densities of closed shell atoms, although their definition of $e_{x}$ differs from ours. In most gradient expansions, the Laplacian terms allowed by symmetry are transformed into $|\nabla n(\mathbf{r})|^{2}$ terms via an integration by parts. This is possible only when the dependence on $l$ is linear, however, which is not the case here, as can be seen from Fig. 3b. Furthermore, the integration by parts destroys the physical interpretation in terms of the $\mathrm{XC}$ hole and so hinders further progress. We note that there is a one-to-one relationship between position and $l$ in each of our systems, and so an enhancement factor of the form $F_{x}(l)$ might not be as universal as Fig. $3 \mathrm{~b}$ suggests. However, the consistent results obtained for all three systems, the energetic significance of the Laplacian terms, and the strong similarities between the form of the Laplacian and the LDA errors in $e_{\mathrm{xc}}$ (see Fig. 2) give us confidence in the physical importance of the Laplacian in describing inhomogeneity corrections to the LDA.

We thank R. Q. Hood for useful discussions, G. Rajagopal and A.J. Williamson for help with the VMC codes, and L. Smith for help with visualization. M.N. was supported by an EU Marie Curie Fellowship under Grant No. ERBFMBICT961736. Our calculations were performed on the CRAY-T3E at EPCC.

*Present address: Complexity Research Group, Admin2 PP5, British Telecom Laboratories, Martlesham Heath, Suffolk IP5 3RE, UK.
[1] P. Hohenberg and W. Kohn, Phys. Rev. 136, B864 (1964); W. Kohn and L. J. Sham, Phys. Rev. 140, A1133 (1965).

[2] J. P. Perdew, K. Burke, and M. Ernzerhof, Phys. Rev. Lett. 77, 3865 (1996); J. P. Perdew and Y. Wang, Phys. Rev. B 33, 8800 (1986).

[3] A. D. Becke, Phys. Rev. A 38, 3098 (1988); A. D. Becke, J. Chem. Phys. 98, 5648 (1993).

[4] C. Lee, W. Yang, and R. G. Parr, Phys. Rev. B 37, 785 (1988).

[5] B. L. Hammond and W. A. Lester, Jr., and P. J. Reynolds, Monte Carlo Methods in Ab Initio Quantum Chemistry (World Scientific, Singapore, 1994).

[6] E. Engel and S. H. Vosko, Phys. Rev. B 50, 10498 (1994); C. J. Umrigar and X. Gonze, Phys. Rev. A 50, 3827 (1994); A. D. Becke, J. Chem. Phys. 109, 2092 (1998).

[7] R. G. Parr and W. Yang, Density Functional Theory of Atoms and Molecules (Oxford University Press, Oxford, 1988).

[8] Hartree atomic units with $e=m=4 \pi \epsilon_{0}=\hbar=1$ are used throughout.

[9] M. Nekovee et al., Adv. Quantum Chem. 33, 189 (1999).

[10] R. Q. Hood et al., Phys. Rev. Lett. 78, 3350 (1997).

[11] C. J. Umrigar, K. G. Wilson, and J. W. Wilkins, Phys. Rev. Lett. 60, 1719 (1988); A. J. Williamson et al., Phys. Rev. B 53, 9640 (1996).

[12] At $\lambda=1$ this procedure might be viewed as a VMC realization of Hohenberg and Kohn's first theorem: given the exact ground-state density, we find the corresponding ground-state many-body wave function.

[13] L. M. Fraser et al., Phys. Rev. B 53, 1814 (1996); A. J. Williamson et al., Phys. Rev. B 55, R4851 (1997).

[14] J. P. Perdew and A. Zunger, Phys. Rev. B 23, 5048 (1981).

[15] M. A. Buijse and E. J. Baerends, in Density Functional Theory of Molecules, Clusters, and Solids, edited by D. E. Ellis (Kluwer Academic Publishers, Dordrecht, 1995).

[16] See, however, Fig. 4 of Ref. [9], where preliminary results containing relatively large finite-size errors $(\sim-0.0003$ a.u. $)$ are shown for $e_{x}^{\mathrm{VMC}}-e_{x}^{\mathrm{LDA}, \mathrm{GGA}}$ and $e_{c}^{\mathrm{VMC}}-e_{c}^{\mathrm{LDA}, \mathrm{GGA}}$.

[17] Y.-H. Kim et al., Phys. Rev. B 61, 5202 (2000); L. Pollack and J. P. Perdew, J. Phys. Condens. Matter 7, 1239 (1999).

[18] J. P. Perdew, S. Kurth, A. Zupan, and P. Blaha, Phys. Rev. Lett. 82, 2544 (1999).

[19] In fact, the GGA corrections have the wrong sign at large $q$, when the fixed-height potential barriers are narrow. In this limit, the electrons tunnel easily from well to well and our systems become more three dimensional.

[20] P. R. T. Schipper, O. V. Gritsenko, and E. J. Baerends, Phys. Rev. A 57, 1729 (1998).

[21] See Levy and Perdew [Phys. Rev. A 32, 2010 (1985)] for a derivation for the case $F_{x}=F_{x}(s)$. The generalization to the case $F_{x}=F_{x}(s, l)$ is straightforward.

[22] For $s=0$, the value of $F_{x}$ is $<1$ on the lower branch of this scatter plot and $>1$ on the upper branch, in contrast to the GGA enhancement factor, which satisfies $F_{x}^{\mathrm{GGA}} \downarrow 1$ as $s \rightarrow 0$. The GGA enhancement factor is constructed such that it approaches the uniform electron gas value of 1 in the limit as $s \rightarrow 0$; the exact $F_{x}$, however, is a function of all density gradients and need not approach 1 when $s=0$ but $l$ is nonzero.

[23] M. Springborg and J. P. Dahl, J. Chem. Phys. 110, 9360 (1999). 